\title{
Magnetophonon resonance in photoluminescence excitation spectra of magnetoexcitons in GaAs/Al0.3Ga0.7As superlattice
}

Dickmann, S.; Tartakovskii, A. I.; Timofeev, V. B.; Zhilin, V. M.; Zeman, J.; Martinez, G.; Hvam, Jørn Märcher

Published in:

Physical Review B Condensed Matter

Link to article, DOI:

10.1103/PhysRevB.62.2743

Publication date:

2000

Document Version

Publisher's PDF, also known as Version of record

Link back to DTU Orbit

Citation $(A P A)$ :

Dickmann, S., Tartakovskii, A. I., Timofeev, V. B., Zhilin, V. M., Zeman, J., Martinez, G., \& Hvam, J. M. (2000). Magnetophonon resonance in photoluminescence excitation spectra of magnetoexcitons in GaAs/Al0.3Ga0.7As superlattice. Physical Review B Condensed Matter, 62(4), 2743-2750.

https://doi.org/10.1103/PhysRevB.62.2743

\section{General rights}

Copyright and moral rights for the publications made accessible in the public portal are retained by the authors and/or other copyright owners and it is a condition of accessing publications that users recognise and abide by the legal requirements associated with these rights.

- Users may download and print one copy of any publication from the public portal for the purpose of private study or research.

- You may not further distribute the material or use it for any profit-making activity or commercial gain

- You may freely distribute the URL identifying the publication in the public portal 


\title{
Magnetophonon resonance in photoluminescence excitation spectra of magnetoexcitons in $\mathrm{GaAs} / \mathrm{Al}_{0.3} \mathrm{Ga}_{0.7} \mathrm{As}$ superlattice
}

\author{
S. Dickmann, A. I. Tartakovskii, V. B. Timofeev, and V. M. Zhilin \\ Institute of Solid State Physics, Russian Academy of Sciences, 142432 Chernogolovka, Moscow District, Russia \\ J. Zeman and G. Martinez \\ Grenoble High Magnetic Field Laboratory, MPI-FKF and CNRS, 38042 Grenoble, Cedex, France
}

J. M. Hvam

Microelectronic Centre, The Technical University of Denmark, DK 2800 Lyngby, Denmark

(Received 14 June 1999; revised manuscript received 28 January 2000)

\begin{abstract}
A strong increase in the intensity of the peaks of excited magnetoexciton (ME) states in the photoluminescence excitation (PLE) spectra recorded for the ground heavy-hole magnetoexcitons (of the $1 s \mathrm{HH}$ type) has been found in a GaAs/ $/ \mathrm{Al}_{0.3} \mathrm{Ga}_{0.7} \mathrm{As}$ superlattice in strong magnetic field $B$ applied normal to the sample layers. While varying $B$, the intensities of the PLE peaks have been measured as functions of energy separation $\Delta E$ between excited ME peaks and the ground state of the system. The resonance profiles have been found to have maxima at $\Delta E_{\max }$ close to the energy of the GaAs LO phonon. However, the value of $\Delta E_{\max }$ depends on quantum numbers of the excited ME state. The revealed very low quantum efficiency of the investigated sample allows us to ascribe the observed resonance to the enhancement of the nonradiative magnetoexciton relaxation rate arising due to LO-phonon emission. The presented theoretical model, being in a good agreement with experimental observations, provides a method to extract $1 \mathrm{sHH}$ magnetoexciton "in-plane" dispersion from the dependence of $\Delta E_{\max }$ on the excited ME state quantum numbers.
\end{abstract}

\section{INTRODUCTION}

Optical transitions in semiconductor superlattices (SL's) have received great attention recently. In contrast to the quasi-two-dimensional (2D) case of quantum wells (QW's), quasiparticles in SL's are not fully confined in the growth direction $\hat{z}$. Moreover, periodicity in the distribution of the materials with different band gaps and elastic constants leads to the formation of minibands in the case of free carriers and excitons, ${ }^{1,2}$ and the appearance of additional optical and acoustic-phonon modes. ${ }^{2-8}$ Numerous investigations concerning optical observation of excitons and magnetoexcitons (ME's) in QW's (e.g., see Refs. 9-12) and in Sl's (Refs. 13-15) usually leave aside the questions related to the nonradiative excitonic relaxation. At the same time, nonradiative excitonic transitions such as phonon emission or absorption allow one to probe indirectly the exciton-energy dispersion $\mathcal{E}(\boldsymbol{k})$ ( $\boldsymbol{k}$ is the exciton wave vector). Furthermore, studies of these processes may provide the only way of the experimental measurement of the excitonic dispersion. Indeed, such a powerful method as the hot-luminescence technique (reported for the first time in Ref. 16), which was widely used for measurements of the hole bandstructure in bulk GaAs ${ }^{17,18}$ and in GaAs/AlGaAs QW's, ${ }^{17,19}$ is inefficient for the study of the excitonic dispersion. Although excited ME states were observed in hot magnetoluminescence measurements, ${ }^{20}$ such experiments can hardly reveal any information on the function $\mathcal{E}(\boldsymbol{k})$. This is because hot-luminescence measurements can only probe excitons in their radiative states, i.e., when $k$ is very small, so that $\mathcal{E}(\boldsymbol{k}) \approx \mathcal{E}(0)$.

The theoretical investigations of excitonic dispersion have attracted large efforts ever since Gor'kov and Dzyaloshinskii's work devoted to three-dimensional ME. ${ }^{21}$ Later, 2D ME was studied in the paper by Lerner and Lozovik ${ }^{22}$ and also in the works concerning 2D excitons without magnetic field. ${ }^{9,10,23}$ Exciton dispersion relations in SL's presenting the dependencies $\mathcal{E}(\boldsymbol{k})$, where $\boldsymbol{k} \| \hat{z}$ (i.e., minibands) were calculated in Ref. 24. In parallel, the excitonic binding energy in SL's were studied theoretically and experimentally in Ref. 25 (see also Refs. 26 and 27), and later the binding energies of ground and excited states in SL's were calculated in magnetic and electric fields. ${ }^{28,29}$ It is worth noting that two dimensionality and strong magnetic fields are the features that usually allow the separation of transverse variables $x$ and $y$ from the longitudinal one $z(\boldsymbol{B} \| \hat{z})$ and in addition permit a simplification of the model for valence band due to the removal of degeneracy. 1,2

The present paper is the result of experimental and theoretical studies on the observation of a resonant behavior in relaxation of ME's in type-I GaAs/ $\mathrm{Al}_{0.3} \mathrm{Ga}_{0.7} \mathrm{As} \mathrm{SL}$ 's in a high-magnetic field perpendicular to the SL layers. In our experiments we detected only photoluminescence (PL) signal from the ground heavy-hole exciton state $1 s \mathrm{HH}$ (we employ the usual notation for $2 \mathrm{D}$ exciton states ${ }^{12,15}$ ) while the energy of the laser excitation was continuously varied in a range of $80 \mathrm{meV}$ above the $1 s \mathrm{HH} \mathrm{PL}$ peak. At particular magnitudes of magnetic field we have observed very strong resonant increases of intensity of peaks corresponding to the excited ME states the in PLE spectra. We interpret this effect as a manifestation of the magnetophonon resonance when strong relaxation of the excited $\mathrm{ME}$ state takes place via longitudinal-optic-(LO) phonon emission. This occurs when frequency $\omega_{\mathrm{LO}}$ is equal to an approximate multiple of the excitonic cyclotron frequency $\omega_{c}$ (a similar effect for free 
electrons in heterojunctions is reported in Refs. 30-32):

$$
\hbar \omega_{\mathrm{LO}}=N \hbar \omega_{c}, \quad N=1,2,3, \ldots
$$

Here $\omega_{c}=e B / \mu c$, where $\mu$ is the reduced excitonic mass for transverse motion in the layer plane. To our best knowledge, this is the first observation of the magnetophonon resonance for excitons, and yet we think the similar phenomenon could manifest not only in SL but in quantum wells and dots as well.

The effect has been found only in one SL sample of the investigated series of SL structures. According to our measurements, the quantum efficiency of this sample is about two orders of magnitude less than that for the other investigated structures. This fact allows us to treat the considered phenomenon as a feature of the enhanced relaxation of the excited ME states rather than the peculiarity of light absorption.

The observed resonance profiles (i.e., integrated intensity of enhanced PLE peaks as a function of their energy separation $\Delta E$ from the ground state) were found to be rather broad (with half-widths above $5 \mathrm{meV}$ ) and have strong maxima at $\Delta E_{\max }$ dependent on the quantum numbers of excited ME states. These facts indicate that transitions to the ground ME state occur via intermediate nonradiative states of the ground excitonic $1 s \mathrm{HH}$ band. The theoretical treatment developed in our paper allows us to extract certain information about the exciton-energy dependence on "in-plane" wave-vector component $\boldsymbol{q}$ from the experimental data.

After the description of the experimental results in Sec. II we present the theoretical study of the phenomenon in Sec. III. Finally, in the discussion of Sec. IV we demonstrate the comparison of the PLE data with the theoretical results and present dispersion curves for the "transverse" $1 s \mathrm{HH}$ band $\boldsymbol{\epsilon}(q) \equiv \mathcal{E}\left(\boldsymbol{q}, k_{z}=0\right)$ extracted from our experiment.

\section{EXPERIMENT}

Samples used in our investigations are molecular-beam epitaxy grown type-I GaAs/ $\mathrm{Al}_{0.3} \mathrm{Ga}_{0.7}$ As SL's. The structures are not intentionally doped and the flat band regime is realized. All the samples have $L_{w}=8$-nm-wide GaAs QW's, while the width of the $\mathrm{Al}_{0.3} \mathrm{Ga}_{0.7}$ As barrier is varied from sample to sample as $L_{b}=2,3,5,10$, and $20 \mathrm{~nm}$. All the SL's consist of 20 periods $d=L_{b}+L_{w}$.

The PLE experiments were performed in a He cryostat with a superconducting magnet providing a magnetic field up to $23 \mathrm{~T}$ normal to the SL layers. A technique based on employing optical fibers was used for the sample excitation and collection of the PLE signal. In the geometry of our experiment the incident laser light propagates in the direction close to the normal to the SL's layers. In order to measure the PLE spectra we tuned a double 1-m monochromator slightly lower or directly to the ground heavy-hole exciton PL peak and scanned the excitation energy of the $\mathrm{Ar}^{+}$-pumped $\mathrm{Ti}$ sapphire laser. The main features of the PLE spectra remained unchanged when the detection position was moved in the limits of the linewidth around the $1 s \mathrm{HH}$ PL peak. PLE of two different polarizations, $\sigma^{+}$and $\sigma^{-}$, was detected by a cooled GaAs detector in the photon counting regime.

Figure 1 displays typical heavy-hole exciton PLE spectra for the SL with $L_{w}=8 \mathrm{~nm}$ and $L_{b}=3 \mathrm{~nm}$ (referred to as

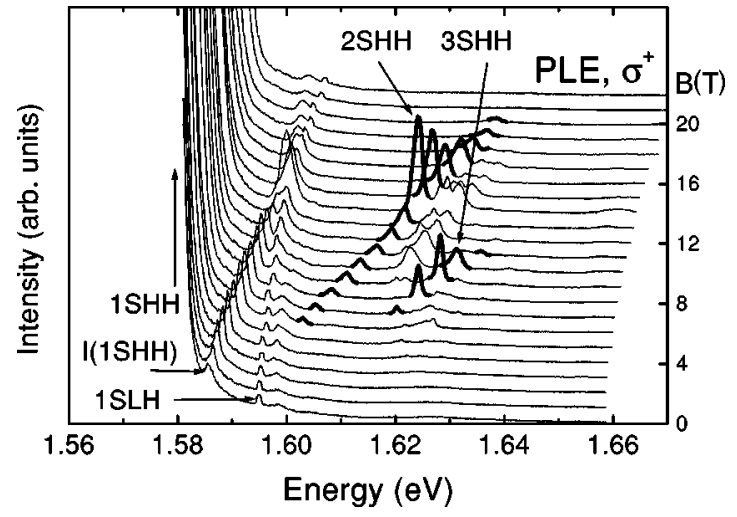

FIG. 1. $\sigma^{+}$PLE spectra recorded for a ground magnetoexciton state for various magnetic fields. The excited magnetoexciton peaks are indicated by arrows.

sample $8 / 3$ below) recorded in magnetic field applied normal to the SL layers. We present here the data for $\sigma^{+}$polarization only, since the observed behavior is very similar to that for $\sigma^{-}$polarization. At $B=0$, the most pronounced peaks in the spectrum are the direct heavy-hole $1 s \mathrm{HH}$ exciton (its position is not clearly resolved in the shown series of spectra because the laser wavelength was scanned from the highenergy side of the $1 s \mathrm{HH}$ line), the indirect heavy-hole $I(1 \mathrm{sHH})$ exciton at $1.586 \mathrm{eV}$ (Ref. 15), and the light-hole $1 s \mathrm{LH}$ exciton at $1.5955 \mathrm{eV} .{ }^{33}$ When $B$ is increased to $6 \mathrm{~T}$, new features become clearly resolved in the spectra above the $1 s \mathrm{LH}$ peak. Energies of these excited states rapidly increase with $B$. The origin of the new peaks can be revealed with the help of an elaborate theoretical analysis of the magnetoexciton band structure. However, this task lies beyond the scope of our investigation. In order to understand the nature of the strongest PLE peaks, we carried out a simplified analysis of PLE spectra recorded at high-magnetic fields. As a result it has been found that the peaks plotted in Fig. 1 by thick lines, correspond to ME's formed by electrons and heavy holes from Landau levels with equal $N=2,3$ $(2 s \mathrm{HH}, 3 s \mathrm{HH}) .{ }^{15}$ In what follows we restrict our investigation to the study of the resonant behavior of these ME states.

As it is seen in Fig. 1, already starting from $B$ of several Teslas the energies of $2 s \mathrm{HH}$ and $3 s \mathrm{HH}$ ME peaks increase quasilinearly with $B$. At $B<6 \mathrm{~T}$, the intensity of the $2 s \mathrm{HH}$ ME peak is weak. Then starting from $B=12$ T the intensity of the $2 s \mathrm{HH}$ peak grows rapidly, reaching its maximum at $B=14 \mathrm{~T}$ and then decreases more slowly. A similar resonant behavior is clearly observed for the $3 s \mathrm{HH} \mathrm{ME}$ at $B \approx 9 \mathrm{~T}$. Similar resonances have been also observed in $\sigma^{-}$polarization, however they occur at slightly larger $B$ : at $B=17 \mathrm{~T}$ for $2 s \mathrm{HH}$ and at $B=9.5 \mathrm{~T}$ for $3 s \mathrm{HH}$.

To summarize the PLE data of Fig. 1 (and of the similar series for $\sigma^{-}$) the integrated intensities of the $2 s \mathrm{HH}$ and $3 s \mathrm{HH}$ ME's are plotted in Figs. 2(a) and 2(b) (black and open squares, respectively) for both polarizations versus the energy separation $\Delta E$ between their positions and the location of the $1 s \mathrm{HH}$ peak. The energy of $1 s \mathrm{HH}$ peak is extracted from another series of PLE measurements. ${ }^{33}$ The variation of $\Delta E$ with increasing magnetic field occurs due to the stronger diamagnetic shifts of the excited ME peaks with respect to that of the $1 s \mathrm{HH}$ line (see insets of Figs. 2(a) and 2(b), where it is seen that $\Delta E$ changes almost linearly with 

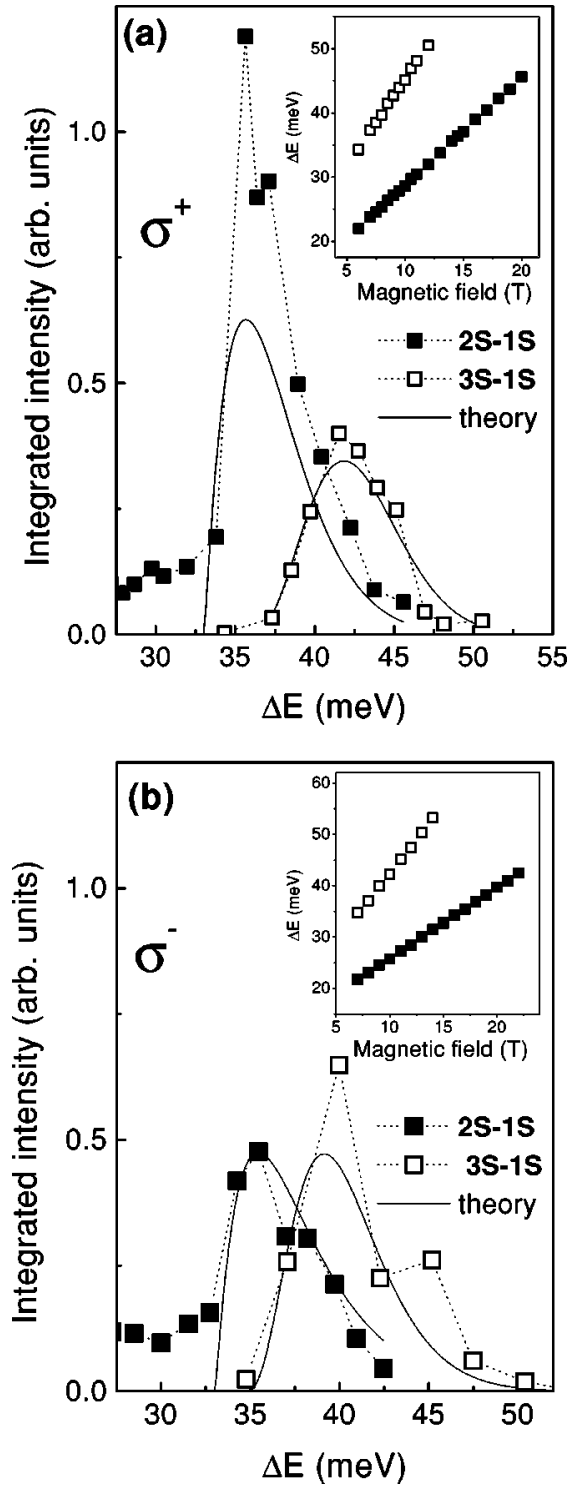

FIG. 2. Integrated intensities of the $2 s \mathrm{HH}$ and $3 s \mathrm{HH}$ PLE peaks for the both polarizations $\left(a-\sigma^{+}, b-\sigma^{-}\right)$as a function of the peak position $\Delta E$ measured from the $1 s \mathrm{HH}$ peak energy. Black and open squares show the experimental data. The insets present the experimental dependencies of $\Delta E(B)$ for the $2 s \mathrm{HH}$ and $3 s \mathrm{HH}$ peaks.

the magnetic field both for $2 s \mathrm{HH}$ and $3 s \mathrm{HH}$ ME's). Figures 2(a) and 2(b) show that a very strong increase (by a factor of $10-20)$ of ME peak intensities arises when $\Delta E \approx 35$ and $\approx 40 \mathrm{meV}$ for the $2 s \mathrm{HH}$ and $3 s \mathrm{HH}$ peaks, respectively. These values are very close to the energy of optical phonons in $\mathrm{GaAs} / \mathrm{Al}_{x} \mathrm{Ga}_{1-x} \mathrm{As} .{ }^{5}$ Note however that the resonance for the $2 s \mathrm{HH}$ peak appears at smaller $\Delta E$ than that for the $3 s \mathrm{HH}$ peak. At the same time the resonant enhancement for the $2 s \rightarrow 1 s$ transition naturally occurs in stronger magnetic field than the resonance for the $3 s \rightarrow 1 s$ one.

The following features of the resonances should be noted as well: (i) their decay as a function of $\Delta E$ is slower than their buildup; (ii) a structure is observed at $\Delta E \approx 37 \mathrm{meV}$ for $2 s \mathrm{HH} \mathrm{ME}$ and at $\Delta E \approx 45 \mathrm{meV}$ for the $3 s \mathrm{HH}$ state.

As it is mentioned above, the precise comparative experiments showed that sample $8 / 3$ had quantum efficiency $\eta$ about two orders of magnitude lower than those of all other SL samples investigated. Meanwhile, other features characterizing the quality of the samples such as PL linewidths and Stokes shifts in PLE spectra are very similar for all samples (1.5-2 $\mathrm{meV}$ and 1-1.5 meV, respectively). Moreover, the heavy-hole exciton binding energy was found to decrease continuously from the sample with $L_{b}=20 \mathrm{~nm}$ to the sample with $L_{b}=2 \mathrm{~nm}$ without any peculiarity for the structure with $L_{b}=3 \mathrm{~nm} .{ }^{15}$ These two facts imply that the band structure of sample $8 / 3$ in the energy range close to the energy of the superlattice ground state is as yet unperturbed. We can suppose that strong nonradiative recombination channels are most likely due to deep trapping centers, which originate from native lattice defects. ${ }^{34}$ However, the nature of nonradiative centers that is caused by growth procedure details of a particular sample plays no significant role in our paper.

Closing this section we would like to note that the intensity of PLE peaks would reflect the absorption efficiency only in the case of $\eta=1$. This condition together with the condition of carriers radiative lifetime being long compared with their relaxation time would permit the excited ME's to relax into the ME ground state without scattering into other states that relax later nonradiatively. On the other hand, in our case of $\eta \ll 1$, the intensity of some peaks can be resonantly enhanced by the mechanism that strongly reduces the relaxation time of the ME transition from the excited state to the radiative ground state and hence decreases to some extent the probability of nonradiative ME escape.

\section{THEORY}

\section{A. Qualitative consideration of the transition and the formulation of the problem}

First note that both states, namely, initial $|i\rangle$, which is $2 s \mathrm{HH}$ or $3 s \mathrm{HH}$, and final $\left|f_{0}\right\rangle=1 s \mathrm{HH}$ have very small exciton wave vectors, since $|i\rangle$ arises by virtue of direct light absorption, whereas $f_{0}$ relaxation directly provides the optical PLE signal. More accurately if $\boldsymbol{q}=\left(k_{x}, k_{y}\right)$ and $k_{z}$ are the transverse and longitudinal components, then for these states we find for the used experimental geometry that

$$
q \lesssim 10^{4} \mathrm{~cm}^{-1}, \quad k_{z} \lesssim 10^{5} \mathrm{~cm}^{-1} .
$$

Here the right-hand sides are determined by the homogeneity breakdown due to impurities or by momenta of absorbed and emitted photons. Meanwhile the actual extent of the exciton wave functions is of the order of $10 \mathrm{~nm}$, since, it is determined by three values: the magnetic length $l_{B}=(c \hbar / e B)^{1 / 2}$, the effective exciton Bohr radius $a_{0}=\hbar^{2} \varepsilon_{0} / \mu e^{2}$, and the period $d$. In the scale of inverse lengths this reads

$$
k_{0} \sim 2 \pi / l_{B} \sim 2 \pi / a_{0} \sim 2 \pi / d \sim 10^{6}-10^{7} \mathrm{~cm}^{-1} .
$$

The significant difference between the values (3.1) and (3.2) allows one to conclude that the considered resonant transition $i \rightarrow f_{0}$ is an indirect one. Indeed, due to momentum conservation, in a direct transition the emitted optical phonon would have a negligibly small wave vector (3.1). As a result the macroscopic LO polarization field applied to ME may be considered to be homogeneous, and in the limit $k / k_{0} \rightarrow 0$ the corresponding transition matrix element would be simply proportional to $\left|\left\langle i|\boldsymbol{r}| f_{0}\right\rangle\right|$ (with $\boldsymbol{r}=\boldsymbol{r}_{2}-\boldsymbol{r}_{1}$ being the difference between electron and hole positions), which turns out to be 


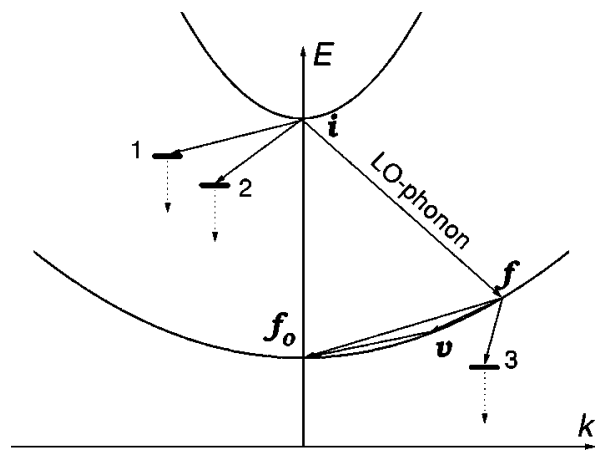

FIG. 3. Diagram of possible transitions. LO-phonon-emission $i$ $\rightarrow f$ gives rise to the studied effect. Transitions $i \rightarrow 1, i \rightarrow 2$, and $f$ $\rightarrow 3$, lead to nonradiative exciton annihilation. Transitions $i \rightarrow f$ $\rightarrow f_{0}$ and $i \rightarrow f \rightarrow v \rightarrow f_{0}$ are the examples of nonradiative 2(or 3 ) $s \mathrm{HH} \rightarrow 1 s \mathrm{HH}$ relaxation yielding the measured luminescence signal.

equal to zero because of the identical symmetry of the initial and final states with respect to inversion. Thus we consider the LO transition not right into $\left|f_{0}\right\rangle$ but initially into $|f\rangle$, which is a $1 s \mathrm{HH}$ exciton state with the wave vector $k \sim k_{0}$. Finally, the transition $f \rightarrow f_{0}$ is a nonradiative process, e.g., provided by acoustic-phonon emission.

The assumed transitions are schematically demonstrated in Fig. 3. The transitions resulting in nonradiative exciton relaxation are also shown in this diagram. One can see that in the assumed scheme the PLE signal is proportional to the rate of the allowed LO-phonon emission.

Among the others the diagram reflects one essential simplification used in our calculations presented below: we ignore the broadening of the exciton peaks, which naturally occurs due to crystal and SL imperfections (the curves in Fig. 3 have zero widths). The diagram implies that $\Delta E=E_{i}$ $-E_{f_{0}}$ should be larger than $\hbar \omega_{\mathrm{LO}}$. However, in the experiment the beginning of the enhanced relaxation for $2 s$ exciton occurs even at $\Delta E \approx 33 \mathrm{meV}$ (see Fig. 2), which is lower than the LO-phonon energy in a bulk GaAs crystal $\approx 36 \mathrm{meV}$. Here we should note that the value of $\Delta E$ (extracted from PLE spectra) corresponds to the separation of the excited and ground-state exciton absorption maxima. Meanwhile the density-of-states in the vicinity of the ground state is presented by a rather wideband, and at low temperatures the emission comes mostly from the lower states. This fact provides an effective Stokes shift; so that the resonant enhancement of the ground-state luminescence due to LOphonon mediated relaxation may start at $\Delta E \approx \hbar \omega_{\mathrm{LO}}-\Delta_{\mathrm{PL}}$ $-\Delta_{\mathrm{St}}$, where the PL linewidth $\Delta_{\mathrm{PL}} \approx 2 \mathrm{meV}$ and the Stokes shift $\Delta_{\mathrm{LO}} \approx 1 \mathrm{meV}$. Both values $\Delta_{\mathrm{PL}}$ and $\Delta_{\mathrm{St}}$ are determined by disorder effects.

In order to describe the data presented in Fig. 2 in the approximation of zero exciton-level width we have to employ an effective energy of LO phonon that is lower than the tabulated bulk value. As it was described above this disagreement can be easily eliminated by the consideration of the finite exciton peak widths. However, for the simplicity of the model this procedure is omitted in our calculations. The deviation of the LO-phonon energy from the bulk value can also be related to an inevitable effective averaging of the SL multimode phonon spectrum in the single-mode approach employed in our model. Later in Sec. IV we will briefly discuss the role of the multimode spectrum in the context of the phenomenon studied.

Thus our approach should be considered as a theoretical model, which simplifies the analytical calculation of the relaxation rate as follows:

(i) results are obtained in the strong magnetic-field approximation, which enables us to separate the transverse and longitudinal variables.

(ii) we consider only the heavy-hole band ignoring its nonparabolicity and the difference in the effective hole masses in $\mathrm{GaAs}$ and $\mathrm{Al}_{0.3} \mathrm{Ga}_{0.7} \mathrm{As}$ layers, though we take into account the anisotropy of effective hole mass.

(iii) we also ignore the difference of the effective electron masses in GaAs and $\mathrm{Al}_{0.3} \mathrm{Ga}_{0.7}$ As layers.

(iv) we consider only one LO-phonon mode with the effective energy $\hbar \omega_{0}=33 \mathrm{meV}$ independent of phonon wavevector direction (to avoid the possible misunderstandings we replace everywhere below $\omega_{\mathrm{LO}}$ by $\omega_{0}$ ). Besides, as in the case of bulk GaAs, ${ }^{35}$ we use only the Fröhlich-type Hamiltonian for electron-LO-phonon as well as for hole-LOphonon interactions (cf. Refs. 2 and 36).

(v) we ignore possible momentum conservation breakdown and finite widths of the excitonic peaks that occur due to random impurity potential or quantum well and interface roughness.

(vi) finally, any spin-orbit terms in the used Hamiltonians are disregarded, therefore the presented theory does not take into consideration the effects of "fine structure", in the PLE signal dependent on light polarization (such effects can be rather peculiar, see, e.g., Ref. 12).

In spite of these essential assumptions we believe that such a simplified approach accounts for the most important aspects of the transition shown in Fig. 3 and yields reliable information about the ME relaxation rate.

According to Fig. 3 a considerable enhancement takes place when the intermediate state $|f\rangle$ is a real (not virtual) state of the lowest excitonic band. The general formula for the total probability of the LO-phonon emission is

$$
W_{\mathrm{LO}}=\sum_{f} W_{i f}
$$

where $W_{i f}$ is the probability of the transition into the state $|f\rangle$. Meanwhile subsequent processes of relaxation $f \rightarrow f_{0}$ are not the matter of our interest here.

Evidently the energy conservation leads to the equations

$$
E_{i}(B)-E_{0}(B)=\hbar \omega_{0}+\mathcal{E}(\boldsymbol{k}), \quad E_{f}-E_{0}=\mathcal{E}(\boldsymbol{k}) .
$$

Here the intermediate exciton state energy is written as $E_{f}$ $=E_{0}+\mathcal{E}(\boldsymbol{k})$ where $E_{0}=E_{f_{0}}$ is the ground-state energy and $\mathcal{E}(\boldsymbol{k})$ is the excitonic kinetic energy. The left side in the first Eq. (3.4) depends quasilinearly on $B$ [cf. the insets in Figs. 2(a) and 2(b)] because $E_{i}-E_{0} \equiv N \hbar \omega_{c}+\delta U(B)$, where $\delta U$, being much less than $\hbar \omega_{c}$, is the difference of binding energies in $|i\rangle$ and $\left|f_{0}\right\rangle$ states.

At the same time we will see that in strong magnetic field the matrix element for the $i \rightarrow f$ transition has a rather sharp maximum in the vicinity of $q=q_{m} \sim l_{B}^{-1}$, which provides really the resonant dependence of the PLE signal on the magnetic field. 
Let us employ the same material parameters as in Refs. 29 and 24 , i.e., for the transverse (in the layer of the well) and for the longitudinal hole masses we get $m_{h \perp}^{*}=0.18 m_{e}$ and $m_{h \|}^{*}=0.34 m_{e}$, respectively. The electron mass is $m_{e \perp}^{*}=m_{e \|}^{*}$ $=0.067 m_{e}$, and the dielectric constant $\varepsilon_{0}$ is equal to 12.5 . Then the "transverse" excitonic Bohr radius $a_{0}$ $=\hbar^{2} \varepsilon_{0} / \mu e^{2}$ is $14 \mathrm{~nm}$, and for the actual magnetic fields we obtain $l_{B} \leqslant L_{w}<a_{0}$. This fact justifies the employment of the strong magnetic-field approximation: $l_{B} \ll a=\min \left(a_{0}, L_{w}\right)(a$ is the characteristic distance between an electron and a hole in the $\hat{z}$ direction).

The specific dependence $\mathcal{E}(\boldsymbol{k})$ is unknown. Nevertheless, the calculations for "free" exciton ${ }^{21}$ in a strong magnetic field and for the exciton in SL with $L_{w}=L_{b}$ and $B=0$ (Ref. 24) make it possible to estimate this value and to find that $\partial \mathcal{E} / \partial q \sim \mu e^{4} l_{B}^{2} q / \varepsilon_{0}^{2} \hbar^{2} \gg \partial \mathcal{E} / \partial k_{z}$. Indeed according to Ref. 24 the miniband width for a SL with $L_{w}=L_{b}=3 \mathrm{~nm}$ is approximately $6 \mathrm{meV}$. Consequently for our SL with the same $L_{b}$ and with $L_{w}=8 \mathrm{~nm}$, the miniband width should be smaller than $1 \mathrm{meV} .{ }^{37}$ In a strong perpendicular magnetic field this value is to be even more strongly reduced and thus it becomes negligible in comparison with the expected characteristic energy of dependence $\epsilon(q)$.

Further, the summation in Eq. (3.3) leads to the result that contains the density of allowed states $|f\rangle$. This value is inversely proportional to the Jacobian of the change from variables of integration over phase space to the integration over $f$-exciton energy and over $k_{z}$ wave-vector component:

$$
\frac{\partial\left(\mathcal{E}, k_{z}\right)}{\partial\left(q, k_{z}\right)} \approx d \epsilon / d q .
$$

The transition probabilities in Eq. (3.3) are expressed in terms of the relevant matrix element $\mathcal{M}_{i \rightarrow f}$,

$$
W_{i f}=\frac{2 \pi}{\hbar}\left|\mathcal{M}_{i \rightarrow f}\right|^{2} \delta\left(E_{i}-E_{f}-\hbar \omega_{0}\right)
$$

which in their turn is calculated using the wave functions of the excitonic states.

\section{B. Excitonic wave functions}

We can write the excitonic wave functions in the following manner (cf. Refs. 21 and 22):

$$
\begin{aligned}
\Psi\left(\boldsymbol{r}_{1}, \boldsymbol{r}_{2}, z_{1}, z_{2}\right)= & \mathcal{L}^{-1} \exp \left(i \boldsymbol{R} \boldsymbol{q}+\frac{i}{2 l_{B}^{2}}\left[\boldsymbol{r}_{1} \times \boldsymbol{r}_{2}\right] \boldsymbol{B} / B\right) \\
& \times \Phi\left(\boldsymbol{r}-\boldsymbol{r}_{0}\right) F\left(z_{1}, z_{2}\right) .
\end{aligned}
$$

Here $j=1,2$ denotes electron and hole, $\boldsymbol{r}_{j}=\left(x_{j}, y_{j}\right)$ is the 2D vector, $\boldsymbol{r}=\boldsymbol{r}_{1}-\boldsymbol{r}_{2}, \quad \boldsymbol{R}=\left(\boldsymbol{r}_{1}+\boldsymbol{r}_{2}\right) / 2, \quad \boldsymbol{r}_{0}=\boldsymbol{B} \times \boldsymbol{q} l_{B}^{2} / B$, and $\mathcal{L}$ is the sample size in the plane $(\hat{x}, \hat{y}) . \Phi(\boldsymbol{r})$ obeys the twodimensional Schrödinger equation in the main approximation of which the Coulomb interaction can be neglected. ${ }^{21,22}$ In this case $\Phi(\boldsymbol{r}) \approx|N, m, \boldsymbol{r}\rangle$ ( $N$ is the Landau-level number, $m$ is the magnetic quantum number), where

$$
\begin{aligned}
|N, m, \boldsymbol{r}\rangle= & {\left[\frac{N !}{2^{|m|+1}(N+|m|) ! \pi}\right]^{1 / 2} r^{|m|} l_{B}^{-|m|-1} } \\
& \times L_{N}^{|m|}\left(r^{2} / 2 l_{B}^{2}\right) e^{i m \varphi-r^{2} / 4 l_{B}^{2}}
\end{aligned}
$$

( $L_{N}^{m}$ are Laguerre polynomials). The energies corresponding to these functions are

$$
E_{N m}^{(0)}=\hbar \omega_{c}[N+1 / 2(|m|+\gamma m+1)],
$$

where $\gamma=\left(m_{h \perp}^{*}-m_{e}^{*}\right) /\left(m_{h \perp}^{*}+m_{e}^{*}\right)$.

In the next approximation Coulomb interaction can be taken into account with the use of the operator

$$
\mathcal{H}_{\text {int }}=e^{2} / \varepsilon_{0} \sqrt{\left(\boldsymbol{r}+\boldsymbol{r}_{0}\right)^{2}+w^{2}}, \quad \text { where } \quad w=z_{1}-z_{2}
$$

in perturbation theory. ${ }^{21,22}$ Coulomb interaction in function $F\left(z_{1}, z_{2}\right)$ should be included from the very first step. The Bloch theorem for this function takes place if one changes the variables; namely, if

$$
\begin{gathered}
\mathcal{F}_{k_{z}}(Z, w)=F\left(Z+\gamma_{1} w, Z-\gamma_{2} w\right), \quad \text { where } \\
\gamma_{1}=m_{h \|}^{*} / M, \quad \gamma_{2}=m_{e}^{*} / M,
\end{gathered}
$$

then

$$
\begin{aligned}
\mathcal{F}_{k_{z}}(Z, w) & =\mathcal{L}_{z}^{-1 / 2} e^{i k_{z} Z} v_{k_{z}}(Z, w), \quad \text { where } \quad v_{k_{z}}(Z+d, w) \\
& =v_{k_{z}}(Z, w)
\end{aligned}
$$

( $\mathcal{L}_{z}$ is the sample size along $\hat{z}$ ). We restrict ourselves to the one-band approximation assuming for all $i$ and $f$ states that $v_{k_{z}}(Z, w)$ presents the ground-state function of the twoparticle motion in the $\hat{z}$ direction. This function can evidently be normalized so that

$$
\int_{-\infty}^{+\infty} \int_{Z_{0}}^{Z_{0}+d}\left|v_{k_{z}}(Z, w)\right|^{2} d Z d w=d .
$$

\section{Matrix element calculation and inverse transition time}

Optical phonons in SL's have considerable energy dependence on their wave-vector direction. Moreover, for arbitrary direction the classification of the optical phonon branches as longitudinal and transverse is impossible due to the inhomogeneity of the superlattice medium along the $z$ axis. We choose the simplified model and calculate $\mathcal{M}_{i \rightarrow f}$ employing the Hamiltonian of exciton-LO-phonon interaction in the following form: ${ }^{36}$

$$
\begin{aligned}
\mathcal{H}_{\mathrm{opt}}= & \frac{1}{\mathcal{L}}\left(\frac{\hbar}{\mathcal{L}_{z}}\right)^{1 / 2} e^{-i \omega_{0} t} \sum_{\boldsymbol{k}} U_{\mathrm{opt}}(\boldsymbol{k})\left(e^{i \boldsymbol{q} \boldsymbol{r}_{1}+i k_{z} z_{1}}-e^{i \boldsymbol{q} \boldsymbol{r}_{2}+i k_{z} z_{2}}\right) \\
& + \text { H.c.. }
\end{aligned}
$$

Final results include only the squared modulus of the vertex, which is

$$
\left|U_{\text {opt }}\right|^{2}=\frac{2 \pi e^{2} \omega_{0}}{\bar{\varepsilon} k^{2}} .
$$

Here $\bar{\varepsilon}^{-1}=\varepsilon_{\infty}^{-1}-\varepsilon_{0}^{-1}$ (the standard notations ${ }^{35}$ are used). 
Note that the calculation of $\left\langle i\left|\mathcal{H}_{\text {opt }}\right| f\right\rangle$ with the functions (3.8) without Coulomb interaction gives exactly zero in the result. Indeed the factorization in the form of the product of one-particle $\mathrm{N}$-Landau-level functions is always possible for these functions. Therefore if we are interested in the transition between the different levels $N_{1}$ and $N_{2}$, then the matrix element of a one-particle operator always includes the convolution over the transverse variables $\boldsymbol{r}_{j}$ of one of the particles $\left\langle N_{1}, m \mid m, N_{2}\right\rangle$ which is zero because $N_{1} \neq N_{2}$. Thus $\mathcal{M}_{i \rightarrow f}$ is defined by Coulomb corrections to $\Phi(\boldsymbol{r})$, which were discussed above. Taking into account this comment, the final expression for the matrix element is

$$
\begin{aligned}
\mathcal{M}_{i \rightarrow f}= & \sum_{v} \frac{\left\langle v,\left.\boldsymbol{k}\right|_{\perp}\left\langle f, \boldsymbol{k}\left|\mathcal{H}_{\mathrm{int}}\right| v, \boldsymbol{k}\right\rangle_{\perp}\left|\mathcal{H}_{\mathrm{opt}}\right| i, 0\right\rangle}{E_{i}^{(0)}-E_{v}^{(0)}-\hbar \omega_{0}} \\
& +\sum_{v} \frac{\left\langle f, \boldsymbol{k}\left|\mathcal{H}_{\mathrm{opt}}\right|_{\perp}\left\langle v, 0\left|\mathcal{H}_{\mathrm{int}}\right| i, 0\right\rangle_{\perp} \mid v, 0\right\rangle}{E_{f}^{(0)}-E_{v}^{(0)}+\hbar \omega_{0}} .
\end{aligned}
$$

Here to calculate the expectations one should use the following rules: Brackets ${ }_{\perp}\langle\ldots, \boldsymbol{k}|\ldots| \ldots, \boldsymbol{k}\rangle_{\perp}$ mean the integration over the transverse variables of Coulomb energy with the functions (3.7); if $\boldsymbol{q}=0$ then in Eq. (3.9): $\boldsymbol{r}_{0}=0$. Usual brackets $\langle\ldots\rangle$ mean full expectation (the integration over $\boldsymbol{r}_{1}, z_{1}, \boldsymbol{r}_{2}, z_{2}$ ). Denominators in Eq. (3.14) contain the energy values in zero-order approximation in the interaction and hence one should assume that $E_{i}^{(0)}-E_{f}^{(0)}=N \hbar \omega_{c} \approx \hbar \omega_{0}$. Indicies $i, v$, and $f$ are two dimensional (each of them is a pair $N, m)$. In our case $i=(N, 0), f=(0,0)$. The allowed $v$ in the first sum are $(N, \pm m)$ or $[s, \pm(N-s)]$ with $s=0,1, \ldots, N$ -1 ; and in the second sum $v=(0,0)$. Note also that for $\boldsymbol{q}$ $=0$ the value $(3.14)$ becomes zero.

After all the integrals are calculated, the matrix element to the first order in $l_{B} / a$ takes the form

$$
\mathcal{M}_{i \rightarrow f}=\left(\frac{\hbar}{V}\right)^{1 / 2} \frac{e^{2}}{\hbar \omega_{c} \varepsilon_{0}} U_{\mathrm{opt}} T\left(k_{z}\right) \mathcal{G}_{N}\left(q l_{B}\right),
$$

where $V=\mathcal{L}^{2} \mathcal{L}_{z}$,

$$
\begin{gathered}
T\left(k_{z}\right)=\left\langle\mathcal{F}_{k_{z}}(Z, 0)\left|e^{i k_{z} Z}\right| \mathcal{F}_{0}(Z, 0)\right\rangle=d^{-1} \int_{Z_{0}}^{Z_{0}+d} v_{k_{z}}^{*}(Z, 0) v_{0}(Z, 0) d Z \sim \frac{1}{a}, \\
\mathcal{G}_{1}(p)=-\frac{4 \gamma}{1-\gamma^{2}} e^{-p^{2} / 4}\left(1-e^{-p^{2} / 2}\right)+\sum_{m=1}^{\infty} \frac{\gamma e^{-3 p^{2} / 4} m p^{2 m}\left(m+1-p^{2}\right)}{2^{m}(m+1) !\left[(1+m / 2)^{2}-m^{2} \gamma^{2} / 4\right]}, \\
\mathcal{G}_{2}(p)=\frac{-\gamma\left(1-p^{2}\right)}{1-\gamma^{2}} e^{-p^{2} / 4}\left[1-e^{-p^{2} / 2}\left(1+p^{2} / 2\right)\right]-\frac{2 \gamma}{9-\gamma^{2}} p^{2}\left(1+p^{2} / 4\right) e^{-3 p^{2} / 4} \\
+\sum_{m=1}^{\infty} \frac{\gamma m e^{-3 p^{2} / 4}\left(p^{2} / 2\right)^{m}\left(m+1-p^{2} / 2\right)\left[m^{2}+3\left(1-p^{2} / 2\right)\left(m-p^{2}\right)+2\right]}{2(m+2) !\left[(2+m / 2)^{2}-m^{2} \gamma^{2} / 4\right]} .
\end{gathered}
$$

Now we have to substitute the expression (3.15) into Eq. (3.6). Then in Eq. (3.3) with the help of Eq. (3.5) we change from the summation over states $f$ to the integration over phase space and further to the integration over $\epsilon$ and $k_{z}$. We find then that the $\delta$ function in Eq. (3.6) removes the integration over $\epsilon$. Finally, the result for the total probability of the transition from the excited ME state to some state of the ground ME band is

$$
W_{\mathrm{LO}}(B)=\frac{e^{6} \omega_{0} \Lambda(q) G_{N}\left(q l_{B}\right)}{\left(\hbar \omega_{c}\right)^{2} \varepsilon_{0}^{2} \bar{\varepsilon} d \epsilon / d q},
$$

where

$$
\Lambda(q)=\frac{q}{\pi} \int_{-\infty}^{-\infty} \frac{|T|^{2} d k_{z}}{q^{2}+k_{z}^{2}}
$$

and the functions

$$
G_{N}(p)=\pi \mathcal{G}_{N}^{2}(p)
$$

are plotted in Fig. 4. One should bear in mind that $q$ in Eq. (3.19) is not an independent value but $q=q(B)$ is the root of first Eq. (3.4) with $\mathcal{E}(\boldsymbol{k}) \approx \boldsymbol{\epsilon}(q)$. Therefore $W_{\mathrm{LO}}$ is the func- tion of magnetic field that may be converted to the function of $(N+1) s \mathrm{HH}$-exciton peak position [see insets in Figs. 2(a) and 2(b)].

Note also that the maximum of function $G_{2}$ is shifted substantially to higher $q$ than that of function $G_{1}$. This fact accounts for the smaller experimentally observed $\Delta E_{\max }$ for the $2 s \rightarrow 1 s$ transition with respect to that of the $3 s \rightarrow 1 s$ one.

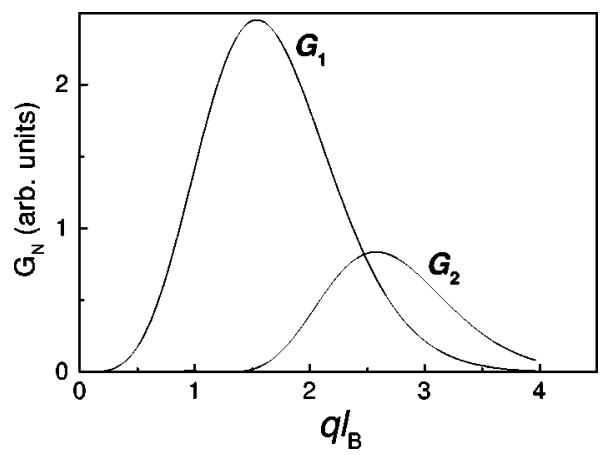

FIG. 4. Functions $G_{N}$ versus the dimensionless "in plane" wave-vector component. Landau level numbers $N=1,2$ correspond to $2 s \rightarrow 1 s$ and $3 s \rightarrow 1 s$ transitions, respectively. 


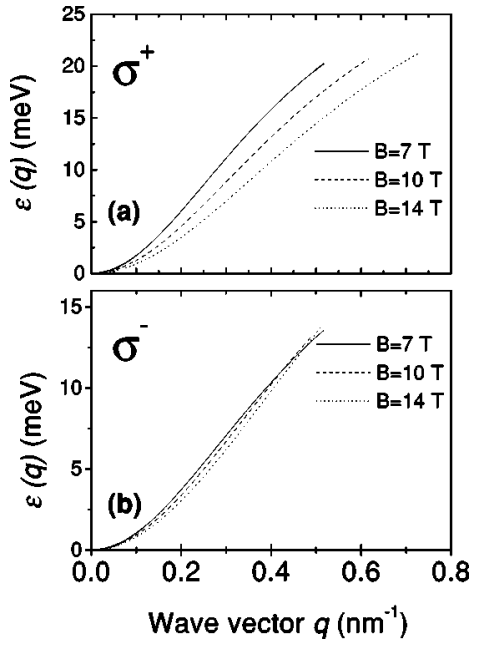

FIG. 5. Transverse energy dispersion functions of Eq. (4.1) when $\mathcal{E}_{b}=5 \mathrm{meV} ; g_{1}^{+}=0.338, g_{2}^{+}=0.019, g_{3}^{+}=0.0561$, for $\sigma^{+}$ polarization (a), and $g_{1}^{-}=-0.054, g_{2}^{-}=0.108, g_{3}^{-}=0.0459$, for $\sigma^{-}$ polarization (b).

Finally one can estimate with the help of Eq. (3.19) the inverse time of relaxation $i \rightarrow f$ in the vicinity of the resonance when $q \simeq q_{N m}$. If $B \simeq 10 \mathrm{~T}, d \epsilon / d q \sim 10^{-5} \mathrm{meV} \mathrm{cm}$ and $|T| \sim 10^{6} \mathrm{~cm}^{-1}$, then this time is

$$
\tau_{N}^{ \pm} \sim W_{\mathrm{LO}}^{-1} \sim 0.01 \mathrm{~ns},
$$

where the superscript + or - labels exciton spin quantum numbers $S_{z}= \pm 1$, which associated with $\sigma^{ \pm}$luminescence polarizations. Generally, the considerable spin-orbit coupling manifests itself in the experimental data, and accordingly we should label by " + " or " - " all the quantities $T, \Lambda, \epsilon$, and $q$ (considered in their turn as the functions of either $B$ or the peak position $\Delta E=E_{i}-E_{0}$ ).

\section{DISCUSSION}

The intensity of the PLE signal under the resonant conditions should be proportional to the inverse time (3.22). Nevertheless, an immediate comparison with experimental data of Figs. 2(a) and 2(b) is impossible as long as the functions $\epsilon_{ \pm}(q)$ are unknown. The alternative approach is to find these functions being guided by this comparison. Note that the following results for $\epsilon_{ \pm}$obtained below are rather qualitative and should be considered as an estimate of the energy dependence on the component $q$.

Let us specify the form of energy dispersion phenomenologically as

$$
\epsilon_{ \pm}(q)=\mathcal{E}_{b} \frac{\left(g_{1}^{ \pm}+g_{2}^{ \pm} B^{1 / 2}\right)\left(q l_{B}\right)^{2}}{1+g_{3}^{ \pm}\left(q l_{B}\right)^{2}},
$$

where $\mathcal{E}_{b}$ is a parameter of the order of the exciton binding energy ${ }^{25,28}$ and $B$ is measured in Teslas. Naturally the parameters $g_{i}^{ \pm}$should be the same for both resonant peaks (that is they are independent of excited exciton quantum number $N$ ), but the set of these parameters varies with the spin quantum number.

The functions (4.1) with $\mathcal{E}_{b}=5 \mathrm{meV}$ are presented in Fig. 5 for the specific sets of $g_{i}^{ \pm}$(indicated in the caption) and for various magnetic fields. Now we can find the values $q_{ \pm}$from

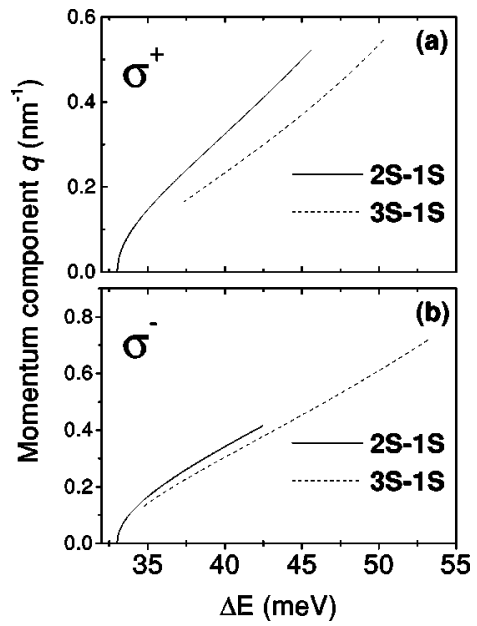

FIG. 6. Values $q^{ \pm}(\Delta E)$ found from the equation $\epsilon_{ \pm}(q)=\Delta E$ $-\hbar \omega_{0}$ for all transitions of $\sigma^{+}$(a) and $\sigma^{-}$(b) polarizations.

Eqs. (3.4) as functions of $\Delta E$ [see Figs. 6(a) and 6(b)]. Meanwhile the dependence $B(\Delta E)$ for magnetic field entering Eq. (4.1) (and also indirectly through $l_{B}$ ) is extracted from the experiment. Therefore, Eqs. (3.4), (3.19), and (4.1) lead to the formula of relevant PLE intensities $I_{N}^{ \pm} \propto W_{\mathrm{LO}}$, namely, in arbitrary units (arb. units) of Figs. 2(a) and 2(b):

$$
I_{N}^{ \pm}(\Delta E)=\frac{C_{ \pm} \mathcal{E}_{b} \Lambda_{ \pm}\left(q_{ \pm}\right)}{B^{3 / 2}\left(\Delta E-\hbar \omega_{0}\right)}\left[q_{ \pm} l_{B}+g_{3}^{ \pm}\left(q_{ \pm} l_{B}\right)^{3}\right] G_{N}\left(q_{ \pm} l_{B}\right) .
$$

Here the additional parameters $C_{ \pm}$arise, which are required for fitting the experimental data presented in arbitrary units. Besides, one should specify the functions $\Lambda_{ \pm}$. We do it with the help of Eq. (3.20) and two-harmonic expansion for the periodic functions $T_{ \pm}\left(k_{z}\right)$,

$$
T_{ \pm} \propto 1+h_{1}^{ \pm} \cos \left(k_{z} d\right)+h_{2}^{ \pm} \cos \left(2 k_{z} d\right) .
$$

The solid lines in Figs. 2(a) and 2(b) correspond to the dependencies (4.2) with $C_{+}=6.1$ arb. units $\times \mathrm{T}^{3 / 2}$ and $C_{-}$ $=10.6$ arb units $\times \mathrm{T}^{3 / 2}$. In our calculations we use $h_{1}^{+}$ $=1.68, h_{2}^{+}=0.902, h_{1}^{-}=1.79, h_{2}^{-}=1.34$, and the sets of functions $g_{i}^{ \pm}$, which are presented in Fig. 5. These parameters are found during the fitting of the experimental points of 2(a) and 2(b). Meanwhile the picture of such a comparison remains qualitatively the same even in the case when $h_{1}^{ \pm}$ $=h_{2}^{ \pm}=0$. It is also important that all the optimum parameters $C_{ \pm}, g_{i}^{ \pm}$, and $h_{i}^{ \pm}$turn out to be of the order of 0.1-1. This confirms the validity of the estimate (3.22) and indirectly the choice of the functions (4.1) and (4.3).

Summarizing the results of the paper we see that our theory is in satisfactory agreement with the experimental data. Comparison with experiment leads to reasonable dependencies $\epsilon(q)$ which are presented in Fig. 5. At the same time, a more detailed theory taking into account real multimode phonon spectrum in SL (Refs. 2,6) has yet to be developed. In the real situation we can expect that with changing $\Delta E$ ME relaxation mediated by different dominant opticphonon modes should occur. We think that this change to another type of optic phonons explains the appearance of the shoulders in resonance profiles mentioned in Sec. II. Also one can expect a quasicontinuous increase of the frequency 
of emitted phonons in comparison with the employed parameter $\omega_{0}=33 \mathrm{meV}$ with increasing $\Delta E$. This implies that the real bands $\epsilon(q)$ are more narrow than those calculated in the frame of our model. Actually only the initial portions of the Fig. 5 curves [it seems for $\epsilon(q)<10 \mathrm{meV}$ ] should reflect a real exciton dispersion.

Finally, note that there are other hypothetical ways for the excited ME states to increase their intensity in PLE spectra. First, the increase in the light absorption (not in the ME relaxation) can be caused by a resonant increase in oscillator strengths of the direct radiative transition. This can occur due to the mixing of ME's states with some other quasiparticle states in SL. Second, the increase in the relaxation rate may appear because the intermediate $f$-state overlaps ME states of the region of increased density-of-states, namely, at SL mini- band edge. However, all these opportunities cannot lead to the observed increase in the intensity by a factor of more than 20 times. Moreover, in these cases similar resonances also should be observed in the samples with $L_{b}=2$ and $5 \mathrm{~nm}$ (of course at different magnetic fields), which does not occur.

\section{ACKNOWLEDGMENTS}

The authors thank V. D. Kulakovskii for useful discussions and R. M. Stevenson for the critical reading of the manuscript. This work is supported by Russian Foundation for Basic Research. A. I. T. and V. B. T. thank also INTAS and the Sci.-Technical Program on the Physics of Solid State Nanostructures for the support.
${ }^{1}$ G. Bastard, Wave Mechanics Applied to Semiconductor Heterojunctions (Les Editions de Physique, Les Ulis, 1988).

${ }^{2}$ E. L. Ivchenko and G. E. Pikus, Superlattices and Other Heterostructures: Symmetry and Optical Phenomena (Springer-Verlag, Berlin, 1995).

${ }^{3}$ B. Jusserand, D. Paquet, and A. Regreny, Phys. Rev. B 30, 6245 (1984).

${ }^{4}$ A.K. Sood, J. Menéndez, M. Cardona, and K. Ploog, Phys. Rev. Lett. 54, 2111 (1985); 54, 2115 (1985).

${ }^{5}$ T. Tsuchiya, H. Akera, and T. Ando, Phys. Rev. B 39, 6025 (1989)

${ }^{6}$ J. Menéndez, J. Lumin. 44, 285 (1989).

${ }^{7}$ V.F. Sapega, M.P. Chamberlain, T. Ruf, M. Cardona, D.N. Mirlin, K. Totemeyer, A. Fischer, and K. Eberl, Phys. Rev. B 52, 14 144 (1995).

${ }^{8}$ Yu.A. Pusep, S.W. da Silva, J.C. Galzerani, A.G. Milekhin, V.V. Preobrazhenskii, B.R. Semyagin, and I.I. Marahovka, Phys. Rev. B 52, 2610 (1995).

${ }^{9}$ G. Bastard, E.E. Mendez, L.L. Chang, and L. Esaki, Phys. Rev. 26, 1974 (1982).

${ }^{10}$ R.L. Greene, K.K. Bajaj, and D.E. Phelps, Phys. Rev. B 29, 1807 (1984).

${ }^{11}$ L. Viña, G.E.W. Bauer, M. Potemski, J.C. Maan, E.E. Mendez, and W.I. Wang, Phys. Rev. B 41, 10767 (1990).

${ }^{12}$ M. Bayer, T.L. Reinecke, S.N. Walck, V.B. Timofeev, and A. Forchel, Phys. Rev. B 58, 9648 (1998).

${ }^{13}$ K. Fujiwara, K. Kawashima, T. Yamamoto, N. Sano, R. Cingolani, H.T. Grahn, and K. Ploog, Phys. Rev. B 49, 1809 (1994).

${ }^{14}$ V. Mizeikis, D. Birkedal, W. Langbein, V.G. Lyssenko, and J.M. Hvam, Phys. Rev. B 55, 5284 (1997).

${ }^{15}$ A.I. Tartakovskii, V.B. Timofeev, V.G. Lyssenko, D. Birkedal, and J.V. Hvam, Zh. Eksp. Teor. Fiz. 112, 1106 (1997) [JETP 85, 601 (1997)].

${ }^{16}$ D.N. Mirlin, I.Ja. Karlik, L.P. Nikitin, I.I. Reshina, and V.F. Sapega, Solid State Commun. 37, 757 (1981).

${ }^{17}$ T. Ruf, in Phonon Raman Scattering in Semiconductors, Quantum Wells and Superlattices, Vol. 142 of Springer Tracts in Modern Physics (Springer-Verlag, Berlin, 1998), p. 163.

${ }^{18}$ J.A. Kash, Phys. Rev. B 47, 1221 (1993).

${ }^{19}$ J.A. Kash, M. Zachau, M.A. Tischler, and U. Ekenberg, Phys. Rev. Lett. 69, 2260 (1992); M. Zachau, J.A. Kash, and W.T. Masselink, Phys. Rev. B 44, 4048 (1991).
${ }^{20}$ F. Meseguer, F. Calle, C. López, J. M. Calleja, L. Viña, C. Tejedor, and K. Ploog, in Proceedings of the 20th International Conference on the Physics of Semiconductors, edited by E.M. Anastassakis and J.D. Joannopoulos (World Scientific, Singapore, 1990), p. 1461.

${ }^{21}$ L.P. Gor'kov and I.E. Dzyaloshinskii, Zh. Eksp. Teor. Fiz. 53, 717 (1967) [Sov. Phys. JETP 26, 449 (1968)].

${ }^{22}$ I.V. Lerner and Yu.E. Lozovik, Zh. Eksp. Teor. Fiz. 78, 1167 (1980) [Sov. Phys. JETP 51, 588 (1980)].

${ }^{23}$ J.A. Brum and G. Bastard, J. Phys. C 18, L789 (1985).

${ }^{24}$ M.M. Dignam and J.E. Sipe, Phys. Rev. B 41, 2865 (1990).

${ }^{25}$ A. Chomette, B. Lambert, D. Deveaud, F. Clerot, A. Regreny, and G. Bastard, Europhys. Lett. 4, 461 (1987).

${ }^{26}$ H. Chu and Y-C. Chang, Phys. Rev. B 39, 10861 (1989).

${ }^{27}$ A. Chomette, B. Deveaud, F. Clérot, B. Lambert, and A. Regreny, J. Lumin. 44, 265 (1989).

${ }^{28}$ D.M. Whittaker, Phys. Rev. B 41, 3238 (1990).

${ }^{29}$ M.M. Dignam and J.E. Sipe, Phys. Rev. B 43, 4097 (1991).

${ }^{30}$ P. Hawker, A.J. Kent, L.J. Challis, M. Henini, and O.H. Hughes, J. Phys.: Condens. Matter 1, 1153 (1989).

${ }^{31}$ D.J. Barnes, R.J. Nicholas, F.M. Peeters, X-G. Wu, J.T. Devreese, J. Singleton, C.J.G.M. Langerak, J.J. Harris, and C.T. Foxon, Phys. Rev. Lett. 66, 794 (1991).

${ }^{32}$ T.A. Vaughan, R.J. Nicholas, C.J.G.M. Langerak, B.N. Murdin, C.R. Pidgeon, N.J. Mason, and P.J. Walker, Phys. Rev. B 53, 16 481 (1996).

${ }^{33}$ The energy of $1 s \mathrm{HH}$, which we will use in the following, cannot be extracted from this set of spectra but its dependence on magnetic field was obtained from the similar experiment previously (see Ref. 15). In particular, for $B=0$ this energy is $1.5805 \mathrm{meV}$.

${ }^{34}$ For example see D. Wong, H.K. Kim, Z.Q. Fang, T.E. Schlesinger, and A.G. Milnes J. Appl. Phys. 66, 2002 (1989); C.V. Reddy, S. Fung, and C.D. Beling, Phys. Rev. B 54, 11290 (1996).

${ }^{35}$ V.F. Gantmakher and Y.B. Levinson, Carrier Scattaring in Metals and Semiconductors (North-Holland, Amsterdam, 1987).

${ }^{36}$ K.J. Nash and D.J. Mowbray, J. Lumin. 44, 315 (1989).

${ }^{37} \mathrm{An}$ estimate for the miniband width is $\hbar^{2} / M L_{w}^{2} \exp \left(-\alpha L_{b}\right)$, where the factor $\alpha$ characterizes the wave function attenuation in the barrier that depends on the barrier height. If only $L_{w}$ is changed by factor $8 / 3$, we arrive at the indicated estimate. 